\title{
Mechatronik und Telematik in der Viszeralchirurgie
}

\author{
Hubertus Feußner ${ }^{a}$ Peter Michael Schlag ${ }^{b}$ \\ ${ }^{a}$ Chirurgische Klinik und Poliklinik, Klinikum rechts der Isar, Technische Universität München, \\ ${ }^{b}$ Klinik für Chirurgie und Chirurgische Onkologie, Robert-Rössle-Klinik im HELIOS Klinikum Berlin-Buch, \\ Charité-Universitätsmedizin Berlin, Campus Berlin-Buch, Deutschland
}

Mit den Begriffen «Mechatronik» und «Telematik» werden besonders zukunftsträchtige Technologien verbunden, die die wirtschaftliche Entwicklung in Zukunft stimulieren und bestimmen sollen. Eine genaue Beschreibung dessen, was sich hinter diesen Ausdrücken verbirgt, fällt wahrscheinlich schwerer, und erst recht die Übertragung auf mögliche Anwendung in der Viszeralchirurgie.

Sowohl Mechatronik als auch Telematik sind Kunstworte, die aus der Kombination zweier unterschiedlicher Begriffe hervorgegangen sind. Mechatronik entstand aus Mechanik und Elektronik und beschreibt Maschinen- bzw. Gerätesysteme, deren Funktion nicht nur auf der mechanischen Funktion, sondern auch auf der Integration von elektronischen Lösungsansätzen im weitesten Sinne beruht. Telematik wiederum ist ein Kunstwort aus Telekommunikation und Informatik. Telematik in der Medizin wird auch als Telemedizin oder «E-Health» bezeichnet. Entsprechend breit sind auch die Anwendungsfelder der Telematik in der Medizin. Sie reichen von der präoperativen Therapieplanung bis zur Telechirurgie oder der Simulation chirurgischer Eingriffe in der virtuellen Realität.

Die konkrete heutige Bedeutung dieser unterschiedlichen Aspekte von Mechatronik und Telematik in der Viszeralchirurgie ist noch sehr unterschiedlich. Telematikanwendungen haben bereits Einzug in den klinischen Alltag gehalten, während mechatronische Hilfssysteme nach einer Phase der anfänglichen Überschätzung bisher noch kaum praktische Bedeutung erlangen konnten. In den folgenden Artikeln soll eine aktuelle Bestandsaufnahme dieser HighTech-Anwendungen in der Viszeralchirurgie unternommen und gleichzeitig eine Abschätzung der künftigen Entwicklungen gewagt werden. Dabei sind die thematischen Abgrenzungen Navigation, Bildgebung, Robotik, Telemedizin, Simulation sowie Mikround Nanosystemtechnik nur vordergründig. Die Übergänge sind fließend, und bei kaum einer klinischen Anwendung wird ein Themenbereich für sich allein zum Einsatz kommen: Erst das Zusammenwirken der unterschiedlichen mechatronischen und telematischen Technologien ermöglicht weitere Fortschritte.

Damit ist zwangsläufig die Frage «cui bono» verbunden. Was (und wem) nützen diese für sich genommen sicher eindrucksvollen tech- nischen Raffinessen? Der Leser darf zu Recht fordern, dass bereits erreichte oder zumindest zu erwartende Nutzanwendungen in den folgenden Darstellungen ebenfalls aufgezeigt werden. Am ehesten ist dies heute schon für die Telematik möglich, wobei hier exemplarisch die Themenbereiche Telemedizin, präoperative Bildgebung, Navigation und Simulation dargestellt werden.

Im Beitrag von Wilhelm et al. [1] wird deutlich, dass unter anderem durch die Einführung der neuen Gesundheitskarte in Verbindung mit dem neuen Heilberufsausweis in Kürze eine umfassende Vernetzung des Gesundheitswesens erreicht werden wird. Davon werden sicher alle Bereiche der Medizin profitieren, aber die Viszeralchirurgie mit ihrem hohen Anteil an onkologischen Patienten ganz im Besonderen. Telemedizin beschränkt sich aber nicht nur auf die prä- bzw. postoperative Phase. Auch während des eigentlichen Eingriffs kann der Operateur von den Möglichkeiten der Online-Telekonsultation bzw. der Telepräsenz profitieren, in dem er bei Bedarf auch externe Kompetenz sozusagen in den OP holt.

Ähnlich überzeugende Optionen bietet die präoperative Planungsbildgebung, insbesondere in der Leberchirurgie. Erste Daten deuten darauf hin, dass besonders bei komplexeren Resektionsfällen (z. B. erweiterte Hemihepatektomie rechts oder bei zentralen Lebertumoren) die von Lamadé et al. [2] beschriebene Bildgebung eine entscheidende Hilfestellung bei der Therapieplanung geben kann. Es ist zu erwarten, dass diese Möglichkeiten auch bald für die Chirurgie anderer parenchymatöser Organe (z. B. Pankreas) eröffnet werden.

Auch die Navigation wird künftig für die Viszeralchirurgie an Bedeutung gewinnen. Die wissenschaftliche Methodik, zuverlässig zu bestimmen, wo man sich befindet und wie die räumliche Beziehung zu anderen relevanten Objekten ist, stammt ursprünglich aus der Seefahrt. In der Chirurgie und insbesondere der Viszeralchirurgie war die Kunst der Navigation solange kein Gegenstand des Interesses, wie man über einen großen Zugang die Anatomie rasch und übersichtlich freilegen konnte: Das Wissen über die Anatomie zusammen mit dem Einblick in den Situs und der Tastsinn reichten aus, um dorthin zu gelangen, wo eingegriffen werden musste. Seit

\section{KARGER}

Fax +497614520714

(c) 2005 S. Karger GmbH, Freiburg

E-mail Information@Karger.de

www.karger.com
Accessible online at: www.karger.com/cga
Prof. Dr. Hubertus Feußner

Chirurgische Klinik und Poliklinik,Klinikum rechts der Isar

Technische Universität München

Ismaninger Straße 22, 81675 München, Deutschland

Tel. +49 89 41 40-2030, Fax -6030

E-mail feussner@nt1.chir.med.tu-muenchen.de 
aber die Traumaminimierung, z. B. durch Reduktion des Zugangs, zu einem zentralen Anliegen der modernen Chirurgie geworden ist, hat auch die Navigation an Bedeutung gewonnen. Ein Eingriff kann umso zielgerichteter und damit schonender gestaltet werden, je besser die anatomische Situation bekannt ist. Anders als in der Chirurgie des Stütz- und Bewegungsapparats ist die Einführung moderner Navigationsverfahren in der Viszeralchirurgie aber um Größenordnungen schwieriger, da an nichtrigiden Strukturen gearbeitet wird und zudem die in der Orthopädie/Unfallchirurgie und Neurochirurgie verwendeten optoelektronischen Verfahren nicht eingesetzt werden können. In dem Artikel von Kleemann et al. [3] werden die gegenwärtig noch bestehenden Probleme, aber auch die bedeutenden Chancen der Navigationsverfahren geschildert. Mit den vorgenannten telematischen Modulen der schnellen Datenverarbeitung, Bildverarbeitung und Navigation ist durch die Einbeziehung geeigneter «user interfaces» auch die Simulation chirurgischer - insbesondere minimal invasiver Eingriffe - in der virtuellen Realität möglich. Lehmann et al. [4] zeigen in ihrem Beitrag auf, dass das chirurgische Training in der virtuellen Realität bereits heute sinnvoll eingesetzt werden kann. Aus technischer Sicht sind sicherlich noch Verbesserungen - z. B. beim Grad der Immersion - erforderlich, doch scheinen diese in evolutionären Entwicklungsschritten ohne weiteres erreichbar. Wichtiger ist, dass sich von heute an Chirurgen aktiver in die Gestaltung der Inhalte einbringen, damit in absehbarer Zukunft die heute in der Chirurgie immer noch übliche Vordermannausbildung am Patienten abgelöst werden kann durch ein sorgfältiges, gezieltes Vortraining in einem virtuellen Situs.

Mechatronische Hilfssysteme in der Chirurgie haben immer wieder heftige, kontroverse Diskussionen ausgelöst. Vermeintliche Vorteile von Robotern wie die hohe Präzision, die fehlende Ermüdung usw. wurden euphorisch betont, aber mittlerweile wurde klar, dass diese anscheinend plausiblen Argumente in der klinischen Praxis nicht gelten. Entsprechend zurückhaltend stellen Gutt et al. [5] nach einer umfassenden Darstellung des aktuellen Stands mechatronischer Hilfssysteme deren konkrete heutige Bedeutung dar. Wahrscheinlich sind die heute verfügbaren Systeme nur ein Zwischenschritt auf dem Weg zu einer neuen, computerassistierten Chirurgie.
Noch visionärer sind die potentiellen Optionen für die Viszeralchirurgie aus dem Bereich der Mikro- und Nanosystemtechnik. Clasbrummel et al. [6] deuten die heute fast noch unübersehbaren diagnostischen und therapeutischen Möglichkeiten extrem miniaturisierter Sensoren und Effektoren an und konkretisieren dies am Beispiel eines Blasendrucksensors. Es bedarf nicht allzu viel Phantasie, um ausgehend von diesem Beispiel auch interessante Anwendungsszenarien für die Viszeralchirurgie zu entwerfen, so etwa den Einsatz implantierbarer Sphinkteren, die hochgradig verstümmelnde Eingriffe wie die Rektumexstirpation ablösen können, über die Verwendung von Modulatoren der gastrointestinalen Motilität bei Gastroparese, Kurzdarmsyndrom oder morbider Adipositas bis hin zur minimal invasiven Implantation von artifiziellen parenchymatösen Organen.

So oder so werden Mechatronik und Telematik zusammen mit neuen Biomaterialien, der molekularen Diagnostik und der Molekularbiologie das zukünftige Bild der Viszeralchirurgie dramatisch verändern. Die konventionelle Chirurgie hat bereits jetzt überall dort an Bedeutung verloren, wo weniger invasive therapeutische Optionen zur Verfügung stehen - sei es in Form erfolgreicher konservativ-medikamentöser Verfahren wie bei der Ulkuskrankheit oder in Form neuer interventioneller Verfahren wie bei der TIPPSImplantation zur Behandlung der portalen Hypertension.

Dagegen konnte die Chirurgie überall dort wieder «Boden gewinnen», wo es durch Einführung neuer - insbesondere minimal invasiver - Verfahren gelang, das therapeutische Ziel schonender als bisher zu erreichen, Beispiele hierfür sind die chirurgische Behandlung des Gallensteinleidens und der Refluxkrankheit. Die Herausforderung besteht darin, weitere innovative Interventionsverfahren zu entwickeln, die die chirurgischen Therapiemöglichkeiten verbessern oder es möglich machen, Krankheitsentitäten chirurgisch zu behandeln, für die bisher noch keine operative Behandlung in Betracht kam. Dieses Ziel zu erreichen wird überhaupt nur dann möglich sein, wenn auch in der Viszeralchirurgie konsequent moderne Technologie genutzt wird. Deshalb sollte auch der praktisch tätige Viszeralchirurg das Potential neuer Technologien zumindest im Ansatz kennen, um die eventuelle Nutzung im eigenen Fachgebiet in Betracht ziehen zu können.

\section{Literatur}

1 Wilhelm D, Weber H, Feußner H: Telemedizin. Chir Gastroenterol 2005;21(suppl 2):1-6.

2 Lamadé W, Ulmer C, Ritter F, Pleitgen H-O, Otto G, Thon K-P: Virtuelle Operationsplanung: Aktueller Stand und Perspektiven. Chir Gastroenterol 2005;21(suppl 2):7-13.

3 Kleemann M, Hildebrand P, Mirow L, Roblick U,
Bürk C, Bruch HP: Navigation in der Viszeralchirurgie. Chir Gastroenterol 2005;21(suppl 2):14-20.

4 Lehmann KS, Buhr HJ, Ritz J-P: Virtuelle chirurgische Operationssimulation. Chir Gastroenterol 2005;21(suppl 2):21-28.

5 Gutt CN, Reiter MA, Koeninger J, Choudhry A, Mehrabi A, Büchler MW: Robotik in der Viszeral- chirurgie. Chir Gastroenterol 2005;21 (suppl 2): 29-37.

6 Clasbrummel B, Muhr G, Jettkant B, Dehm J, Schlierf R, Mokwa W, Möllendorf G: Mikro- und Nanosystemtechnik - medizintechnologische Aspekte am Beispiel des Blasendrucksensors. Chir Gastroenterol 2005;21(suppl 2):38-42. 